\title{
Adenylosuccinate lyase enhances aggressiveness of endometrial cancer by increasing killer cell lectin-like receptor C3 expression by fumarate
}

\author{
Haengki Park ${ }^{1} \cdot K_{\text {Kenji Ohshima }}{ }^{1}$ Satoshi Nojima ${ }^{1} \cdot$ Shinichiro Tahara $^{1} \cdot$ Masako Kurashige $^{1} \cdot$ Yumiko Hori $^{1}$. \\ Daisuke Okuzaki $\mathbb{D i D}^{2} \cdot$ Naoki Wada ${ }^{1} \cdot$ Jun-ichiro Ikeda ${ }^{1} \cdot$ Eiichi Morii $^{1}$
}

Received: 6 July 2017 / Revised: 5 December 2017 / Accepted: 5 December 2017

(c) United States \& Canadian Academy of Pathology 2018

\begin{abstract}
Adenylosuccinate lyase (ADSL) is an enzyme that plays important roles in de novo purine synthesis. Although ADSL was reported to be upregulated in various malignancies, such as colorectal, breast, and prostate cancer, as well as gliomas, the mechanism by which elevated ADSL expression contributes to cancer has not been elucidated. We previously performed a shotgun proteomics analysis to characterize specific proteins associated with the properties of the aldehyde dehydrogenase (ALDH)-high cell population, which was reported to be involved in tumorigenic potential, and showed that ADSL expression is upregulated in the ALDH-high population of endometrial cancer. Here, we showed that ADSL is involved in endometrial cancer aggressiveness by regulating expression of killer cell lectin-like receptor C3 (KLRC3), which is a receptor expressed on natural killer cells. Immunohistochemical analysis indicated that ADSL expression increased as endometrioid carcinoma specimens became more poorly differentiated and higher degree of primary tumor progression. Knockdown of ADSL in endometrial cancer cells decreased cell proliferation, migration, and invasive capability, and caused the cells to adopt a more rounded shape. DNA microarray analysis and quantitative real-time PCR showed that KLRC3 expression was decreased in ADSL knockdown cells. Knockdown of KLRC3 in endometrial cancer cells resulted in the same phenotype as knockdown of ADSL. Moreover, fumarate, which could be produced by ADSL and was recently shown to be an oncometabolite, recovered KLRC3 expression in ADSL knockdown cells, suggesting that fumarate produced by ADSL could regulate KLRC3 expression. Our findings indicate that ADSL enhances cell proliferation, migration, and invasive capability through regulation of KLRC3 expression by fumarate.
\end{abstract}

\section{Introduction}

Endometrial cancer is one of the most common malignancies of the female genital system [1]. We reported previously that aldehyde dehydrogenase 1 (ALDH1), a

Haengki Park and Kenji Ohshima contributed equally to this work.

Electronic supplementary material The online version of this article (https://doi.org/10.1038/s41374-017-0017-0) contains supplementary material, which is available to authorized users.

Eiichi Mori

morii@molpath.med.osaka-u.ac.jp

1 Department of Pathology, Osaka University Graduate School of Medicine, Suita, Osaka, Japan

2 Genome Information Research Center, Research Institute for Microbial Diseases, Osaka University, Suita, Osaka, Japan potential marker of normal and malignant stem cells [2-7], was related to tumorigenic potential and invasive capability in endometrial cancer [8, 9]. In our previous study, adenylosuccinate lyase (ADSL) was shown to be among the proteins that were highly expressed in the ALDH-high cell population of the endometrial cancer cell line, HEC1B [9].

ADSL is an enzyme that is involved in de novo purine synthesis [10] and has been reported to be upregulated in several malignancies, including colorectal, breast and prostate cancer, and gliomas [11-13]. In addition, ADSL expression was shown to be increased in non-differentiated colorectal cancer and melanoma cells in vitro [14-16]. However, the mechanism by which elevated ADSL expression contributes to the cancerous properties of these cells has not been elucidated. Moreover, there have been no reports regarding an association between ADSL expression and endometrial cancer. 
ADSL also produces fumarate through the two catalytic reactions: the conversion of succinylaminoimidazole carboxamide ribotide (SAICAR) to aminoimidazole carboxamide ribotide (AICAR) and fumarate, and the conversion of adenylosuccinate (also called succinyladenosine monophosphate, S-AMP) to adenosine monophosphate (AMP) and fumarate. Fumarate was recently shown to be an oncometabolite, accumulating in fumarate hydratasedeficient cells and activating oncogenic pathways [17-20]. Fumarate has been shown to induce epigenetic changes by inhibiting $\alpha$-ketoglutarate-dependent dioxygenases that are involved in DNA and histone demethylation [21, 22]. Recently, it was reported that fumarate accumulation suppresses microRNA (miR)-200 transcription and induces the epithelial-to-mesenchymal transition (EMT) by inhibiting ten-eleven translocation (TET)-mediated demethylation [23]. However, whether fumarate produced by ADSL affects cancer cell properties has not been determined.

Killer cell lectin-like receptor C3 (KLRC3) has been described as a member of the natural killer group 2 (NKG2) group, which is expressed in natural killer cells. However, its precise function has not been elucidated. Recently, it was reported that KLRC3 forms a complex with CD94 and DAP12 intracellularly, and not on the cell surface [24]. Other than natural killer cells, it was reported that KLRC3 was expressed in glioblastoma cells and was associated with cell proliferation, migration, clonogenicity, and tumorigenesis [25]. These results suggested that the KLRC3/DAP12 complex may interact with intracytoplasmic signaling pathways.

Here, we present a novel pathological role of ADSL in endometrial cancer. Silencing of ADSL in endometrial cancer cells decreased cell proliferation, migration, and invasive capability by reducing KLRC 3 expression. Moreover, fumarate recovered KLRC3 expression in ADSL knockdown cells, suggesting that fumarate produced by ADSL could regulate KLRC3 expression. Our results indicate that ADSL, KLRC3, and fumarate are important for the aggressiveness of endometrial cancer cells.

\section{Materials and methods}

\section{Antibodies and reagents}

Antibodies were obtained from the following sources: mouse monoclonal antibody to ADSL (ab119013) was from Abcam (Cambridge, UK); rabbit monoclonal antibodies to Akt (\#4691), phospho-Akt (Ser473) (\#4060), and $\beta$-actin horseradish peroxidase (HRP-conjugated) (\#5125) were from Cell Signaling Technology (Danvers, MA, USA); and anti-mouse (\#330) and anti-rabbit (\#458) secondary antibodies were from Medical \& Biological Laboratories
(Nagoya, Japan). Reagents were obtained from the following sources: Dulbecco's modified Eagle's medium (DMEM) was from Wako (Osaka, Japan); fetal bovine serum (FBS) was from Biosera (Kansas City, MO, USA); diethyl fumarate was from Sigma-Aldrich (Milwaukee, WI, USA).

\section{Cell lines and cell culture}

The human endometrial adenocarcinoma cell lines, HEC1B, HEC108, and SNGM, were purchased from the Health Science Research Resources Bank, Osaka, Japan. Cell lines were cultured in DMEM supplemented with $10 \%$ FBS, penicillin $(100 \mathrm{IU} / \mathrm{mL})$, and streptomycin $(100 \mu \mathrm{g} / \mathrm{mL})$ and maintained at $37{ }^{\circ} \mathrm{C}$ in $5 \% \mathrm{CO}_{2}$.

\section{Aldefluor assay and separation of the ALDH-high cell population}

An Aldefluor kit (StemCell Technologies, Vancouver, BC, Canada) was used to isolate the population with high ALDH enzymatic activity. HEC1B, HEC108, and SNGM cells were suspended in Aldefluor assay buffer containing ALDH substrate and incubated for $30 \mathrm{~min}$ at $37^{\circ} \mathrm{C}$. As a negative control, each sample was treated with $50 \mathrm{mmol} / \mathrm{L}$ diethylamino benzaldehyde, a specific ALDH inhibitor. HEC1B, HEC108, and SNGM cells with high ALDH enzymatic activity were sorted by flow cytometry (FACSAria; BD Biosciences, San Jose, CA, USA). In ADSL knockdown experiments, cells were subjected to the Aldefluor assay $72 \mathrm{~h}$ after small interfering RNA (siRNA) transfection.

\section{Patients}

All experimental protocols were approved by the Ethical Review Board of the Graduate School of Medicine, Osaka University (no. 15234), and were performed in accordance with the committee guidelines and regulations. All patients provided informed consent. We examined 100 patients undergoing surgery for endometrioid cancer of the uterine corpus at Osaka University Hospital from 2011 to 2016. Resected specimens were fixed in $10 \%$ formalin and processed for embedding in paraffin. Specimens were stored at room temperature in a dark room. Specimens to be used for evaluation were sectioned at a thickness of $4 \mu \mathrm{m}$ and stained with hematoxylin and eosin.

\section{Immunohistochemistry}

Paraffin-embedded tissues were sectioned, processed, and subjected to immunohistochemistry using a Ventana BenchMark GX (Roche, Basel, Switzerland) with antiADSL antibody (1:100 dilution). The expression of each 
protein was assessed using a visual grading system based on the intensity of staining signals observed with a light microscope. High intensity (score 3), intermediate intensity (score 2), and low intensity (score 1) were defined as strong, medium, and weak staining, respectively. H-scores were assigned using the following formula: $[1 \times(\%$ cells of score 1$]$ $+2 \times(\%$ cells of score 2$)+3 \times(\%$ cells of score 3$)]$. Two pathologists ( $\mathrm{KO}$ and $\mathrm{ST}$ ) scored the results independently. If an inconsistency occurred, a third pathologist (YH) was consulted to achieve consensus.

\section{Immunoblotting}

Cells were rinsed three times with ice-cold phosphate-buffered saline (PBS) and lysed in ice-cold lysis buffer $(1 \%$ NP-40, $10 \mathrm{mM}$ Tris-HCl, $200 \mathrm{mM} \mathrm{NaCl}, 1 \mathrm{mM}$ ethylenediaminetetraacetic acid (EDTA)) containing EDTA-free complete protease inhibitor cocktail (Roche) and PhosSTOP (Roche) for $20 \mathrm{~min}$. The soluble fractions from the cell lysates were isolated by centrifugation at $13,000 \mathrm{rpm}$ for $10 \mathrm{~min}$ in a microcentrifuge. Proteins were analyzed by sodium dodecyl sulfate polyacrylamide gel electrophoresis and immunoblotting following standard protocols using anti-ADSL (1:500 dilution), anti-Akt (1:1000 dilution), anti-phospho-Akt (Ser473) (1:2000 dilution), and anti- $\beta$ actin (HRP-conjugated) antibodies (1:1000 dilution).

\section{siRNA-mediated silencing of ADSL and KLRC3 in HEC1B, HEC108, and SNGM cells}

HEC1B, HEC108, or SNGM cells seeded at $20 \%$ confluence were transfected with ADSL-targeting siRNA (Silencer Select s1133, s1134 and s1135; Thermo Fisher Scientific, Waltham, MA, USA), KLRC3-targeting siRNA (Silencer Select s7890, s7891 and s7892; Thermo Fisher Scientific), or non-targeting control siRNA (AM4611; Thermo Fisher Scientific) using Lipofectamine RNAiMAX Reagent (Thermo Fisher Scientific) at a final concentration of $50 \mathrm{nM}$. The sequences of the ADSL-targeting siRNA were 5'-GGGCAGACAUAUACACGAA-3' (s1133), 5'CAGCUUCUGUGGUUAAGCA-3' (s1134) and 5'-GCC UAUCACAGAUGAACAATT-3' (s1135), and those of the KLRC3-targeting siRNA were 5'-AGACUCAGAUCA UGCUGAA-3' (s7890), 5'-GUCUGCUUUGUAUAGAUA ATT-3' (s7891) and 5'-GCAGCAAAGGAAACCUAAA-3' (s7892).

\section{Microarray analysis}

Total RNAs were extracted from HEC1B cells transfected with siRNA duplex specific for ADSL or non-targeting siRNA $96 \mathrm{~h}$ after transfection using an miRNeasy kit (Qiagen, Hilden, Germany) according to the manufacturer's instructions. Two dye-swapped experiments were performed on a Whole Human Genome Oligo Microarray ver. 2 (G4845A; Agilent Technologies, Boeblingen, Germany) as described previously [26]. The Subio Platform and Subio Basic Plug-in (v1.19; Subio Inc., Aichi, Japan) were used to calculate the fold change between samples with the twotailed Student's $t$-test. $P<0.05$ was taken to indicate statistical significance. Raw data microarray have been deposited in NCBI-GEO under accession numbers GSE100703.

\section{Quantitative real-time PCR}

Total RNA was isolated from cells $96 \mathrm{~h}$ after siRNA transfection using QIAshredder and RNeasy Mini Kit (Qiagen), and reverse transcription was performed using the SuperScript III First-Strand Synthesis System (Thermo Fisher Scientific) according to the manufacturer's instructions. Transcript levels were measured using the StepOnePlus Real-Time PCR system (Applied Biosystems, Foster City, CA, USA). Data were normalized by the expression level of ACTB. Human KLRC3 TaqMan probe and primers (Hs01652462_m1; Applied Biosystems), human TET1 TaqMan probe and primers (Hs00286756_m1; Applied Biosystems), and human ACTB TaqMan probe and primers (Hs01060665_g1; Applied Biosystems) were used. The cells for diethyl fumarate treatment were transfected with ADSL-targeting siRNA or non-targeting control siRNA 24 $\mathrm{h}$ prior to initiation of treatment and then treated with 100 $\mu \mathrm{M}$ diethyl fumarate or dimethyl sulfoxide (DMSO) for 72 $\mathrm{h}$, and total RNA was isolated.

\section{Cell proliferation assay}

Cell proliferation was assayed by the Premix WST-1 Cell Proliferation Assay System (Takara, Shiga, Japan) according to the manufacturer's instructions. The absorbance was measured at 450 and $690 \mathrm{~nm}$ using an SH-9000 lab microplate reader (Hitachi, Tokyo, Japan). Briefly, cells were trypsinized $96 \mathrm{~h}$ after siRNA transfection and $2 \times 10^{3}$ cells were seeded into 96-well plates. The WST-1 assay was conducted $96 \mathrm{~h}$ after seeding.

\section{Cell migration assay}

Cell migration was examined by scratch assay. Cells transfected with siRNA were cultured in 6-cm dishes until they reached $100 \%$ confluence to form a monolayer and a straight line was scraped with a p200 pipette tip. In the diethyl fumarate treatment experiment, the culture media were changed to the ones containing $100 \mu \mathrm{M}$ diethyl fumarate or DMSO after scraping. Multiple fields of view per dish were imaged at the time of scraping and 20 or $16 \mathrm{~h}$ 
after scraping using a BZ-8000 microscope (Keyence, Osaka, Japan). The distances between cells were measured using Image $\mathbf{J}$ software. The percentage of closure was calculated by subtracting the width of the wound at 20 or $16 \mathrm{~h}$ from that at $0 \mathrm{~h}$ and dividing the closing distance by the width of the wound at $0 \mathrm{~h}$.

\section{Cell invasion assay}

Cell invasion was examined using Corning BioCoat Matrigel Invasion Chambers with $8.0 \mu \mathrm{m}$ PET Membrane (Corning, Corning, NY, USA). Aliquots of $5 \times 10^{4}$ cells transfected with siRNA for $96 \mathrm{~h}$ and cultured in serum-free DMEM for $12 \mathrm{~h}$ were seeded in the upper chamber and $10 \%$ FBS-DMEM was added to the lower chamber. After $24 \mathrm{~h}$ of incubation, cells on the top surface of the insert were removed by wiping with a cotton swab. The cells that had invaded the bottom side of the membrane were fixed and stained using Diff-Quik stain (Sysmex, Hyogo, Japan). The numbers of invaded cells were determined by averaging four random fields at $\times 200$ magnification per membrane or counting total cell number per membrane, and the averages of three membranes are presented.

\section{Cell shape analysis}

Cells were imaged using a BZ-8000 microscope $72 \mathrm{~h}$ after siRNA transfection. In the diethyl fumarate treatment experiment, cells were transfected with siRNA $24 \mathrm{~h}$ prior to initiation of treatment, treated with $100 \mu \mathrm{M}$ diethyl fumarate or DMSO for $72 \mathrm{~h}$, and imaged. The circularity of 30 cells was measured using Image $\mathbf{J}$ software. A circularity value of 1.0 indicates a perfect circle, whereas a value approaching 0.0 indicates an increasingly elongated polygon.

\section{Statistical analysis}

A database was created using Microsoft Excel and analyzed using JMP Pro 12 (SAS Institute, Cary, NC, USA). The normality of data was evaluated by Shapiro-Wilk test at a significance level of 0.05 . When normality was confirmed in the groups, the homogeneity of variance was tested by Ftest at a significance level of 0.05 . When two groups were confirmed to be normally distributed with equal variance, Student's $t$-test was applied. When at least one of the two groups was not normally distributed, the Mann-Whitney $U$ test was applied. Assessment of differences between $\mathrm{H}$ scores among endometrioid carcinoma specimens was performed using the Mann-Whitney $U$-test. All other statistical analyses were performed using the Student's $t$-test. Data are shown as mean \pm standard deviation (SD). For all box plots, the box depicts the middle $50 \%$ of the records and the line indicates the median. Statistical significance was set as $P<$ 0.05 (two tailed).

\section{Results}

\section{ALDH-high population of endometrial cancer cells express higher ADSL}

In our previous study, ADSL was included in the top 15 proteins that were highly expressed in the ALDHhigh population of the endometrial cancer cell line HEC1B, compared with the ALDH-low population by a shotgun proteomics analysis based on liquid chromatography-tandem mass spectrometry [9]. To validate this result, we conducted immunoblotting of ADSL in ALDH-high and ALDH-low populations of three endometrial cancer cell lines, HEC1B, HEC108, and SNGM, which were sorted by Aldefluor assay. In all cell lines, the ALDH-high population showed higher levels of expressed ADSL than the ALDH-low population (Fig. 1). This result indicated that the ALDH-high population of endometrial cancer cells actually expresses ADSL at a higher level than the ALDH-low population. On the other hand, the proportion of ALDH-high population was not affected by ADSL knockdown, which indicated that

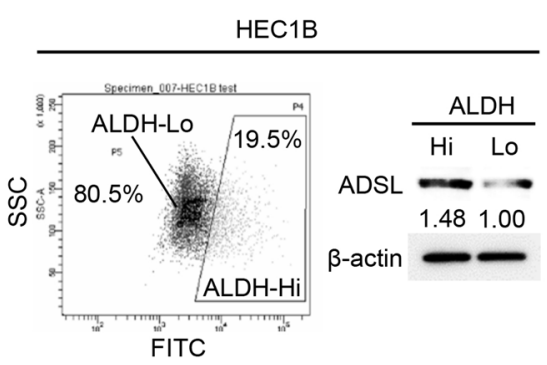

Fig. 1 Adenylosuccinate lyase (ADSL) expression is increased in the aldehyde dehydrogenase (ALDH)-high population. Representative FACS gating strategy for the isolation of ALDH-high (ALDH-Hi) cells and ALDH-low (ALDH-Lo) cells using the Aldefluor assay, and representative immunoblotting of ADSL in sorted ALDH-high and
HEC108
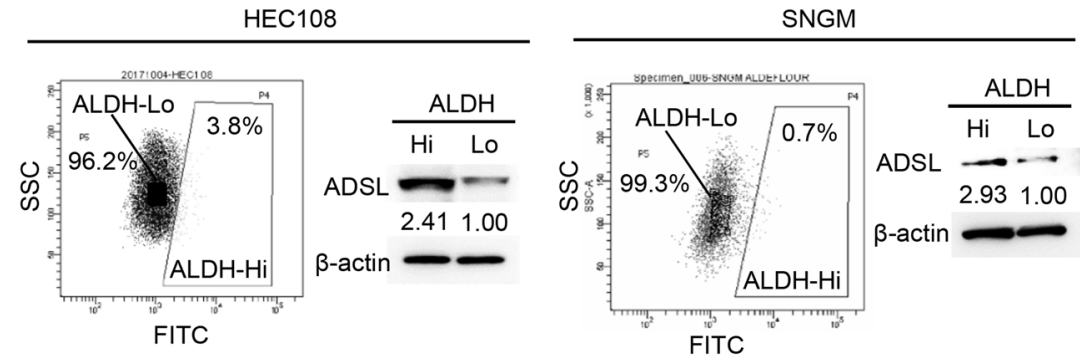

ALDH-low cells. ADSL band intensities of ALDH-Hi cells relative to those of ALDH-Lo cells were calculated after normalization to $\beta$-actin level. All data are representative of two independent experiments 
ADSL might function downstream of ALDH (Supplementary Figure 1).

\section{ADSL expression in human endometrioid carcinoma specimens was correlated with histological aggressiveness and the degree of primary tumor progression}

To examine whether the level of ADSL expression is associated with clinicopathological features of human endometrial cancer, we examined ADSL expression in clinical specimens from 100 cases of endometrioid carcinoma by immunohistochemistry. The clinicopathological characteristics are shown in Table 1. As shown in Fig. 2a, these specimens showed various levels of ADSL expression. The expression levels of ADSL increased as endometrioid carcinoma specimens became more poorly differentiated (Fig. 2b). And also, the expression levels of ADSL were higher in the cases of high degree of primary tumor progression, $\mathrm{T} 2$ and $\mathrm{T} 3$ than in the ones of $\mathrm{T} 1$ (Fig. 2c). The positivity of ADSL expression was not correlated with the degree of lymph node metastasis ( $\mathrm{N}$ grade) (Fig. 2d). These results suggested that the level of ADSL expression is associated with histological aggressiveness

Table 1 The clinicopathological characteristics of the patients

\begin{tabular}{|c|c|}
\hline & $\begin{array}{l}\text { Number } \\
\text { of cases }\end{array}$ \\
\hline \multicolumn{2}{|l|}{ Age (years) } \\
\hline$\geqq 60$ & 43 \\
\hline$<60$ & 57 \\
\hline \multicolumn{2}{|l|}{$\begin{array}{l}\text { Histological } \\
\text { grade }^{\#}\end{array}$} \\
\hline $\begin{array}{c}\text { G1 (well } \\
\text { differentiated) }\end{array}$ & 33 \\
\hline $\begin{array}{l}\text { G2 (moderately } \\
\text { differentiated) }\end{array}$ & 35 \\
\hline $\begin{array}{r}\text { G3 (poorly or } \\
\text { undifferentiated) }\end{array}$ & 32 \\
\hline \multicolumn{2}{|l|}{ T stage* } \\
\hline $\mathrm{T} 1$ & 75 \\
\hline $\mathrm{T} 2 / \mathrm{T} 3$ & 25 \\
\hline \multicolumn{2}{|l|}{ N stage* } \\
\hline N0 & 81 \\
\hline N1 & 19 \\
\hline \multicolumn{2}{|l|}{ M stage* } \\
\hline M0 & 96 \\
\hline M1 & 4 \\
\hline
\end{tabular}

and the degree of primary tumor progression of endometrial cancer.

\section{Silencing of ADSL reduced cell proliferation, migration, and invasive capability}

To examine the mechanism by which ADSL is involved in the aggressiveness of endometrial cancer, we conducted in vitro functional assays of siRNA-mediated ADSL knockdown using the endometrial cancer cell lines HEC1B, HEC108, and SNGM. First, we transfected HEC1B, HEC108, and SNGM cells with three individual siRNA duplexes specific for ADSL (siADSL \#1, \#2, and \#3) or a non-targeting control siRNA (siControl), and confirmed the decrease in ADSL protein expression in ADSL knockdown cells (Fig. 3a). Then, we compared the proliferation of ADSL knockdown and control cells by WST-1 assay. In all HEC1B, HEC108, and SNGM cells, knockdown of ADSL resulted in decreased cell proliferation (Fig. 3b). We next evaluated cell migration and invasive capability by scratch and invasion assays using a Transwell filter system and Matrigel, respectively. Knockdown of ADSL reduced cell migration and invasive capability in all HEC1B, HEC108, and SNGM cells (Figs. 3c, d). Moreover, ADSL knockdown cells showed a more rounded shape than control cells (Fig. 3e). These results indicated that ADSL enhances cell proliferation, migration, and invasive capability, and is also involved in the organization of cell shape.

\section{KLRC3 expression was decreased in ADSL knockdown cells}

Although ADSL is an enzyme involved in de novo purine synthesis, it has been reported that ADSL knockdown did not result in a decrease in the levels of adenine nucleotides (AMP, ADP, and ATP) or GTP [27]. We postulated that the decrease in cell proliferation, migration, and invasive capability by ADSL knockdown in endometrial cancer cells is mediated by a mechanism independent of purine synthesis. To elucidate this mechanism, we performed DNA microarray-based gene expression profiling using ADSL knockdown and control HEC1B cells. In the analysis, 940 of 34,127 probes showed greater than twofold changes in expression $(P<0.05)$. The top 25 genes downregulated in ADSL knockdown HEC1B cells are shown in Table 2. As ADSL was shown to be associated with endometrial cancer cell aggressiveness, we searched for molecules involved in tumor aggressiveness. Among the top 25 molecules, we identified KLRC3, which has been described as a receptor on natural killer cells and was recently shown to be involved in glioblastoma tumorigenesis and aggressiveness [25]. We further examined the function of KLRC3 in 
a

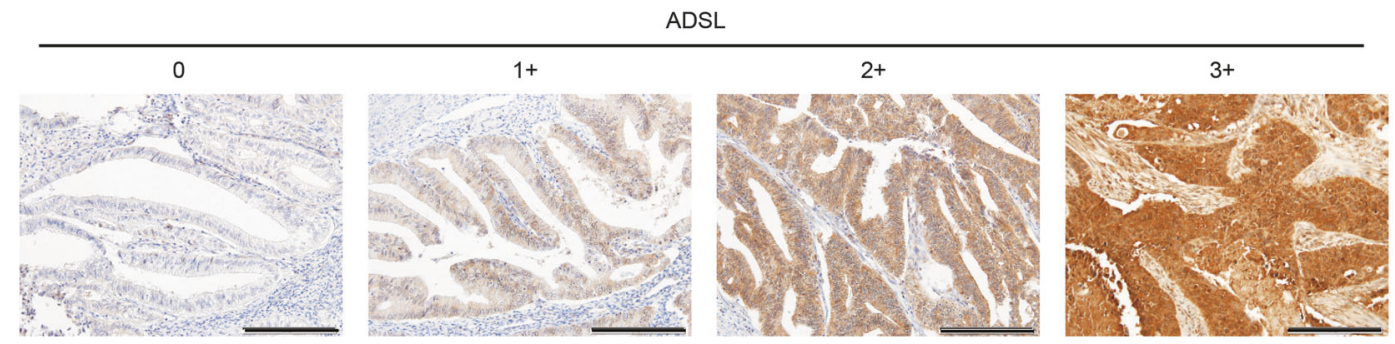

b
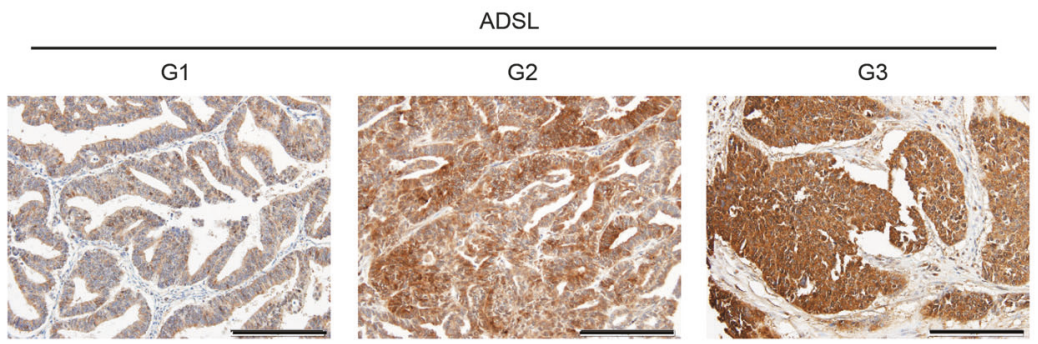

C

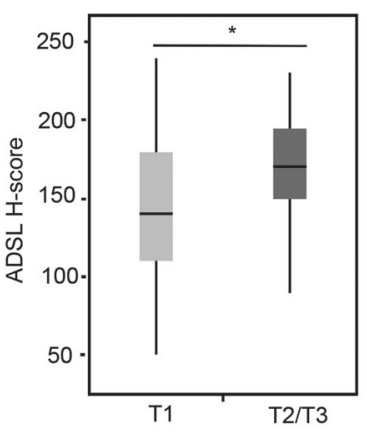

d
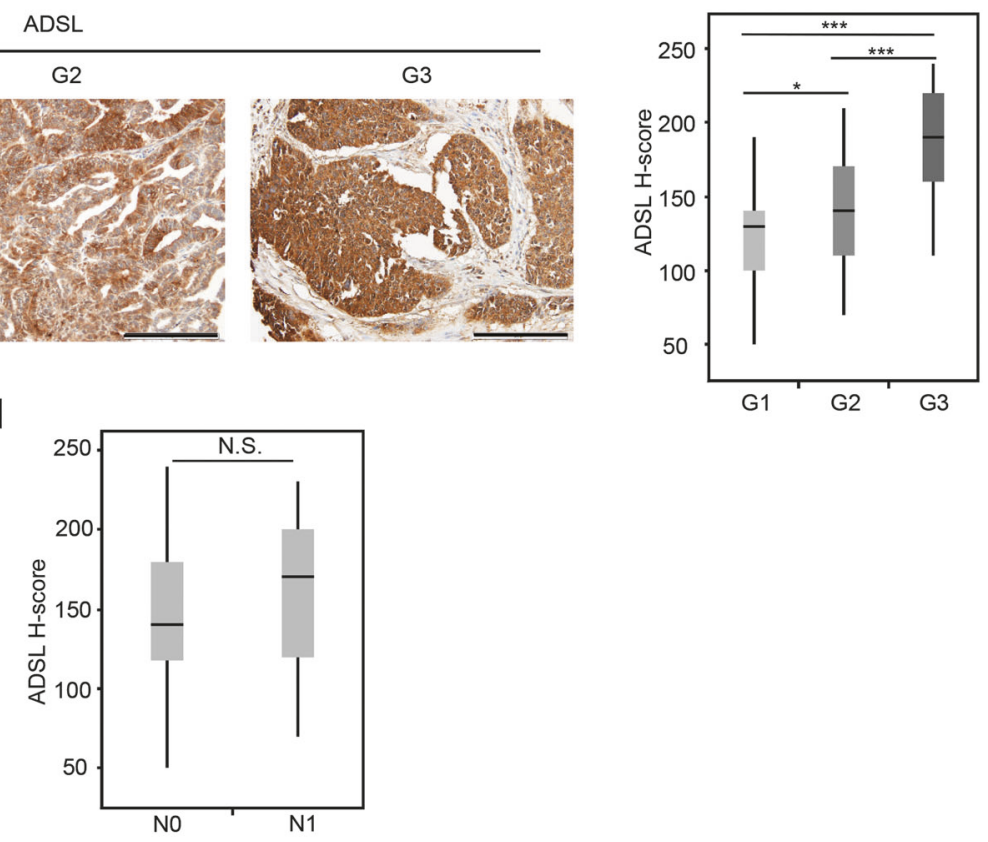

Fig. 2 ADSL expression pattern in clinical specimens derived from human endometrial cancer tissues. a Representative images of immunohistochemistry of ADSL using endometrioid carcinoma tissues with high (score 3), intermediate (score 2), low (score 1), or null (score 0) signal intensity, which were used to calculate $\mathrm{H}$-scores. Scale bars, $200 \mu \mathrm{m}$. b ADSL protein levels in endometrioid carcinoma cases according to histological grade were evaluated by calculating $\mathrm{H}$ scores. Scale bars, $200 \mu \mathrm{m}$. c, d ADSL protein levels in endometrioid

endometrial cancer cells. First, we conducted quantitative real-time PCR of KLRC3 in ADSL knockdown and control cells to validate the results of the microarray analysis. Indeed, ADSL knockdown cells had lower KLRC3 transcript levels than control cells in all cell lines (Fig. 4a). As it has been reported that KLRC3 lacks a specific antibody because an antibody to KLRC3 also binds KLRC1 and KLRC2 with similar affinity [24], we abandoned immunoblotting of KLRC3 in this study. KLRC3 was reported to form a complex with DAP12 intracellularly [24], and DAP12 induces phosphatidylinositol 3-kinase (PI3K)mediated activation of Akt [28-30]. Therefore, we evaluated Akt phosphorylation in ADSL knockdown and control cells by immunoblotting. Indeed, ADSL knockdown cells showed lower Akt phosphorylation than control cells carcinoma cases according to tumor progression ( $\mathrm{T}$ grade) (c) or the degree of lymph node metastasis (N grade) (d) were evaluated by calculating H-scores. The number of examined cases were 100. For box plots, the box depicts the middle $50 \%$ of the records and the line indicates the median. N.S., not significant, $* P<0.05$, $* * * P<0.001$, Mann-Whitney $U$-test

in all HEC1B, HEC108, and SNGM cells (Fig. 4b). These results suggest that reduced expression of KLRC3 by ADSL knockdown leads to decreased Akt phosphorylation, resulting in decreased cell proliferation, migration, and invasive capability of ADSL knockdown cells.

\section{Silencing KLRC3 reduced cell proliferation and migration capability}

To confirm that KLRC3 was actually involved in ADSL knockdown-mediated decreased cell aggressiveness, we conducted in vitro functional assays of siRNA-mediated KLRC3 knockdown. First, we transfected HEC1B, HEC108, and SNGM cells with three individual siRNA duplexes specific for KLRC3 (siKLRC3 \#1, \#2, and \#3), 
Fig. 3 ADSL knockdown decreases cell proliferation, migration, and invasive capability. a Representative immunoblotting of ADSL in HEC1B, HEC108, and SNGM cells transfected with three individual siRNA duplexes specific for ADSL (siADSL \#1, $\# 2$, and \#3) or non-targeting control siRNA (siControl). ADSL band intensities of siADSL cells relative to those of siControl cells were calculated after normalization to $\beta$-actin level. b WST-1 assay of HEC1B, HEC108, and SNGM cells transfected with siADSL or siControl. c HEC1B, HEC108, and SNGM cells transfected with siADSL or siControl were wounded (upper eight pictures). The ratios of the wound closing distance to the width at $0 \mathrm{~h}$ are shown in the lower graphs. $\mathbf{d}$ HEC1B, HEC108, and SNGM cells transfected with siADSL or siControl were subjected to invasion assay for $24 \mathrm{~h}$ (upper four pictures). The numbers of cells invading the membranes are shown in the lower graphs. (e) HEC1B, HEC108, and SNGM cells were imaged $72 \mathrm{~h}$ after transfection of siADSL or siControl (left four pictures). Circularity was measured using Image $\mathbf{J}$ software (right graphs). All data are representative of three independent experiments. Data are shown as means \pm standard deviation (SD) in $\mathbf{b}-\mathbf{d}$. For box plots in e, the box depicts the middle $50 \%$ of the records and the line indicates the median. Scale bars, $400 \mu \mathrm{m}$ in $\mathbf{c}$, $200 \mu \mathrm{m}$ in d, $100 \mu \mathrm{m}$ in e. $* P<$ $0.05, * * P<0.01, * * * P<0.001$, Student's $t$-test

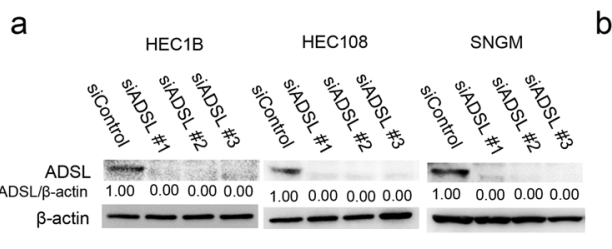

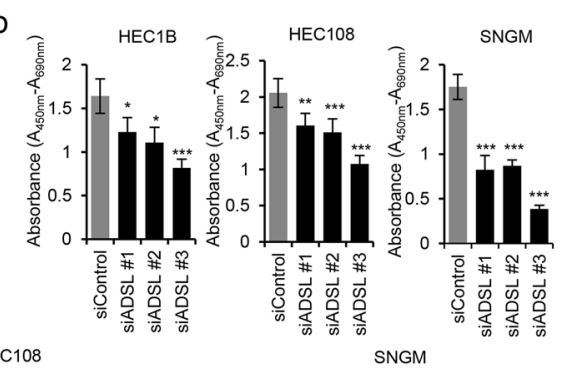

C
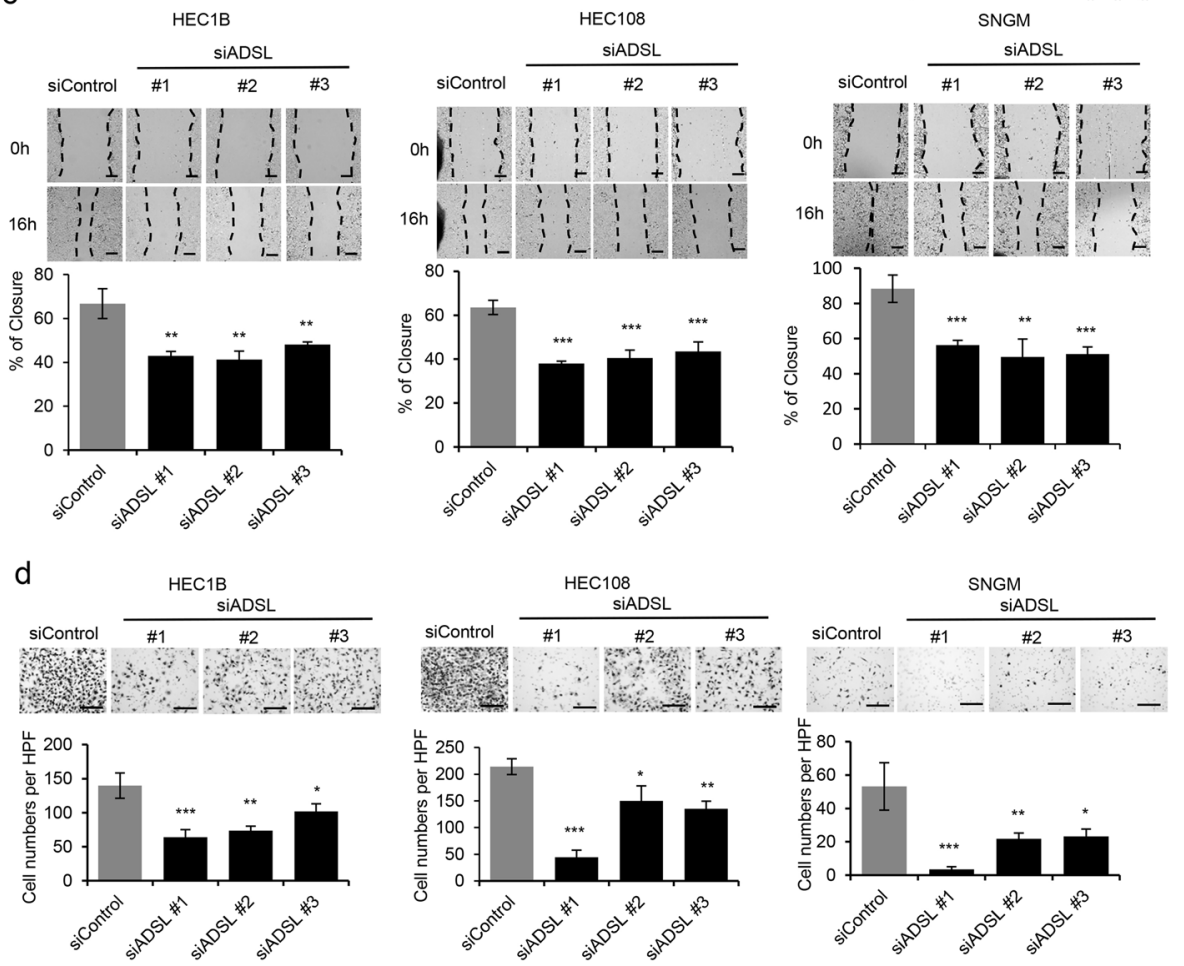

e
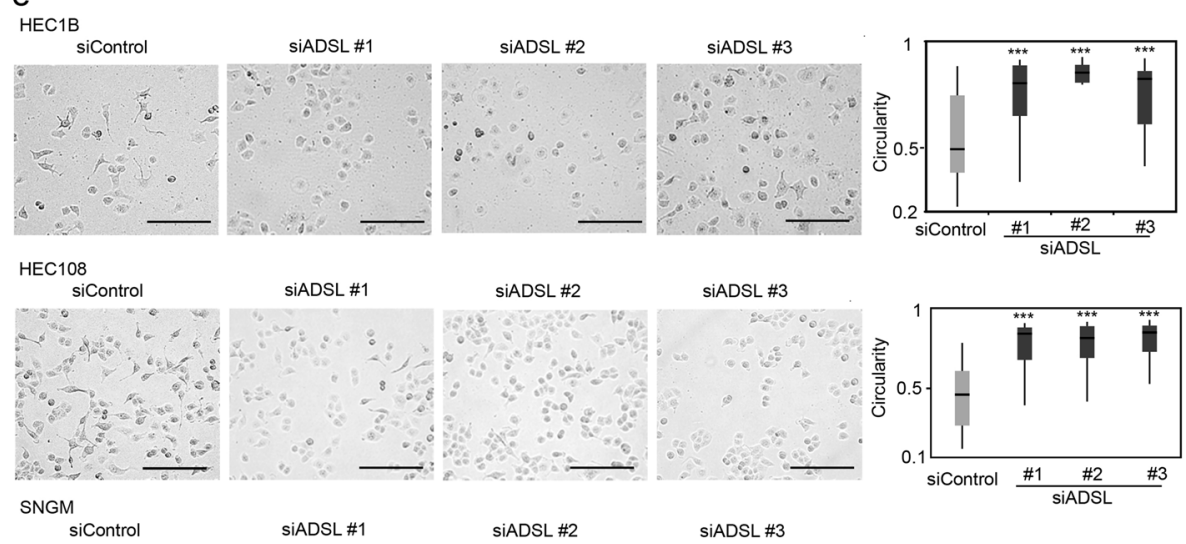

SiADSL \#2

siADSL \#3
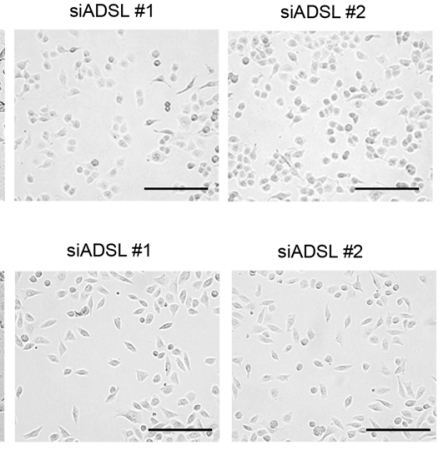

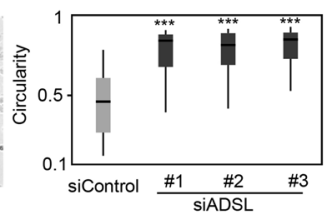

siADSL \#3

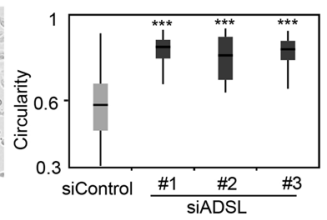

which resulted in $98-72 \%, 92-54 \%$, and $98-62 \%$ decreases in KLRC3 mRNA expression in HEC1B, HEC108, and SNGM cells, respectively (Fig. 5a). Then, we compared proliferation of KLRC3 knockdown and control cells by WST-1 assay. In all HEC1B, HEC108, and SNGM cells, knockdown of KLRC3 resulted in decreased cell 
Table 2 Top 25 genes downregulated in ADSL knockdown HEC1B cells

\begin{tabular}{|c|c|c|}
\hline $\log _{2}$ (fold change) & Gene symbol & Description \\
\hline-4.024 & ADSL & Homo sapiens adenylosuccinate lyase (ADSL), transcript variant 1, mRNA [NM_000026] \\
\hline-3.937 & KLRC4 & Homo sapiens killer cell lectin-like receptor subfamily C, member 4 (KLRC4), mRNA [NM_013431] \\
\hline-3.528 & F2RL2 & Homo sapiens coagulation factor II (thrombin) receptor-like 2 (F2RL2), mRNA [NM_004101] \\
\hline-3.172 & UBD & Homo sapiens ubiquitin D (UBD), mRNA [NM_006398] \\
\hline-3.157 & KLRC3 & $\begin{array}{l}\text { Homo sapiens killer cell lectin-like receptor subfamily C, member } 3 \text { (KLRC3), transcript variant 2, } \\
\text { mRNA [NM_007333] }\end{array}$ \\
\hline-3.002 & BIRC3 & Homo sapiens baculoviral IAP repeat containing 3 (BIRC3), transcript variant 1, mRNA [NM_001165] \\
\hline-2.778 & ANKRD1 & Homo sapiens ankyrin repeat domain 1 (cardiac muscle) (ANKRD1), mRNA [NM_014391] \\
\hline-2.673 & CDRT1 & Homo sapiens CMT1A duplicated region transcript 1 (CDRT1), mRNA [NM_006382] \\
\hline-2.637 & TLR6 & Homo sapiens toll-like receptor 6 (TLR6), mRNA [NM_006068] \\
\hline-2.630 & BHLHE41 & Homo sapiens basic helix-loop-helix family, member e41 (BHLHE41), mRNA [NM_030762] \\
\hline-2.541 & CCL20 & Homo sapiens chemokine (C-C motif) ligand 20 (CCL20), transcript variant 1, mRNA [NM_004591] \\
\hline-2.540 & KPNA7 & Homo sapiens karyopherin alpha 7 (importin alpha 8) (KPNA7), mRNA [NM_001145715] \\
\hline-2.529 & TRIM55 & Homo sapiens tripartite motif containing 55 (TRIM55), transcript variant 2, mRNA [NM_033058] \\
\hline-2.524 & AREG & Homo sapiens amphiregulin (AREG), mRNA [NM_001657] \\
\hline-2.505 & SERPINB8 & $\begin{array}{l}\text { Homo sapiens serpin peptidase inhibitor, clade B (ovalbumin), member } 8 \text { (SERPINB8), transcript variant } \\
\text { 3, mRNA [NM_001031848] }\end{array}$ \\
\hline-2.492 & AREG & Homo sapiens amphiregulin (AREG), mRNA [NM_001657] \\
\hline-2.471 & LTB & $\begin{array}{l}\text { Homo sapiens lymphotoxin beta (TNF superfamily, member 3) (LTB), transcript variant 1, mRNA } \\
\text { [NM_002341] }\end{array}$ \\
\hline-2.422 & ARL14 & Homo sapiens ADP-ribosylation factor-like 14 (ARL14), mRNA [NM_025047] \\
\hline-2.386 & KRT222 & Homo sapiens keratin 222 (KRT222), mRNA [NM_152349] \\
\hline-2.381 & BIK & Homo sapiens BCL2-interacting killer (apoptosis-inducing) (BIK), mRNA [NM_001197] \\
\hline-2.368 & TM4SF19 & $\begin{array}{l}\text { Homo sapiens transmembrane 4 L six family member } 19 \text { (TM4SF19), transcript variant } 1 \text {, mRNA } \\
\text { [NM_138461] }\end{array}$ \\
\hline-2.357 & CXCL3 & Homo sapiens chemokine (C-X-C motif) ligand 3 (CXCL3), mRNA [NM_002090] \\
\hline-2.236 & SYNPO2L & Homo sapiens synaptopodin 2-like (SYNPO2L), transcript variant 2, mRNA [NM_024875] \\
\hline-2.182 & IL32 & Homo sapiens interleukin 32 (IL32), transcript variant 4, mRNA [NM_001012633] \\
\hline-2.178 & SP140 & Homo sapiens SP140 nuclear body protein (SP140), transcript variant 2, mRNA [NM_001005176] \\
\hline
\end{tabular}

proliferation (Fig. 5b). We next evaluated cell migration by scratch assay, and KLRC3 knockdown was shown to result in decreased cell migration (Fig. 5c). In addition, KLRC3 knockdown cells showed a more rounded shape than control cells (Fig. 5d). These results indicated that KLRC3 knockdown in HEC1B, HEC108, and SNGM cells induces the same phenotypes as ADSL knockdown, suggesting that the decreased cell aggressiveness seen with ADSL knockdown was due to reduced expression of KLRC3.

\section{Fumarate recovers KLRC3 expression in ADSL knockdown cells}

ADSL is known to catalyze the two reactions: the conversion of SAICAR to AICAR and fumarate, and the conversion of SAMP to AMP and fumarate (Fig. 6a), and fumarate plays a key role in cell transformation as an oncometabolite, regulating the expression of various genes [20]. We postulated that reduced expression of KLRC3 by ADSL knockdown was due to decreased levels of fumarate in ADSL knockdown cells. To examine this hypothesis, we added diethyl fumarate, which is a cell-permeable form of fumarate, to cells after transfection with siADSL. The results indicated that ADSL knockdown cells treated with diethyl fumarate showed increased expression of KLRC3 in HEC1B, HEC108, and SNGM cells (Fig. 6b). In addition, parallel to the increased expression of KLRC3, treatment with diethyl fumarate restored the cell shape change induced by ADSL knockdown (Fig. 6c). And also, treatment with diethyl fumarate restored cell migration capability reduced by ADSL knockdown (Fig. 6d). These results suggested that ADSL may regulate KLRC3 expression through fumarate production.

\section{Discussion}

Although ADSL expression was reported to be increased in several malignancies, including colorectal, breast, and prostate 


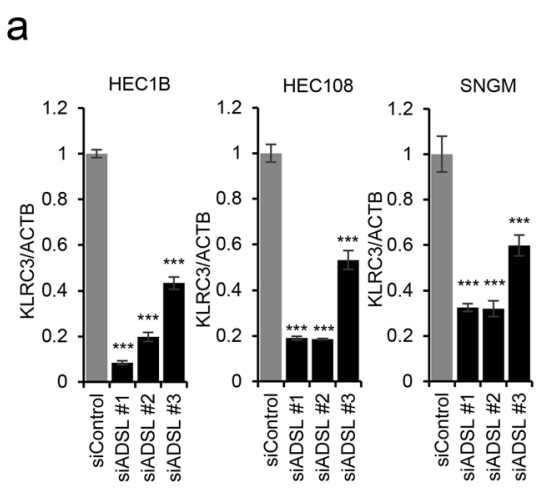

Fig. 4 ADSL knockdown cells show lower KLRC3 levels. a The abundance of $K L R C 3$ mRNA was evaluated by real-time PCR in HEC1B, HEC108, and SNGM cells transfected with siADSL or siControl. Actin beta (ACTB) mRNA abundance was used to normalize real-time PCR results. Data are expressed as a ratio relative to siControl cells. b Representative b

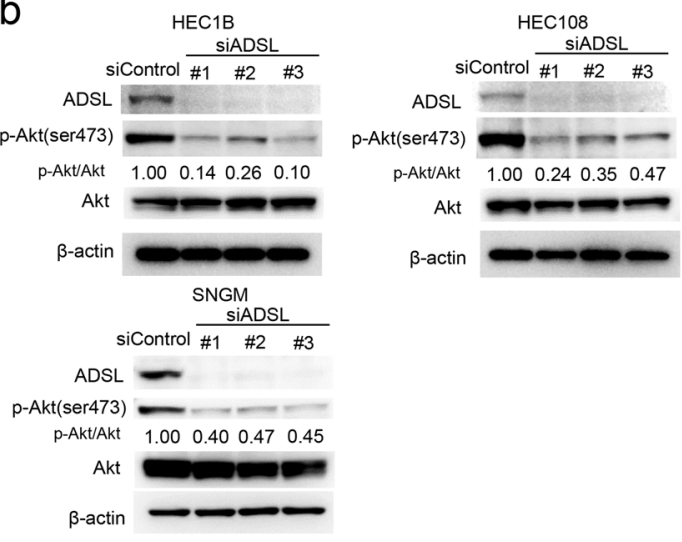

cancer, as well as gliomas [11-13], the mechanism by which increased ADSL expression affects the cancerous properties of the cells has not been elucidated. In this study, we found a novel association between ADSL and tumor cell properties of endometrial cancer. Notably, ADSL was shown to promote tumor cell proliferation, migration, and invasive capability by regulating KLRC3 expression. Moreover, exogenous fumarate recovered KLRC3 expression in ADSL knockdown cells, suggesting that ADSL may regulate KLRC3 expression by fumarate, which is a product of its own activity.

We reported previously that ALDH1 was related to tumorigenic potential and invasive capability in endometrial cancer, and ALDH-high cells showed higher levels of ADSL expression than ALDH-low cells in a shotgun proteomics analysis $[8,9]$. In this study, we conducted immunoblotting of ADSL in ALDH-high and ALDH-low populations sorted by Aldefluor assay, which confirmed that ALDH-high cells actually expressed high levels of ADSL. This result suggested that increased ADSL expression may be one of the mechanisms by which ALDH1 is involved in tumorigenicity and invasive capability in endometrial cancer. Indeed, ADSL expression in human endometrioid carcinoma specimens was correlated with histological aggressiveness and the degree of primary tumor progression.

To examine how ADSL affects tumor cell properties in endometrial cancer, we next conducted in vitro functional assays using ADSL knockdown in the endometrial cancer cell lines HEC1B, HEC108, and SNGM. The ADSL knockdown cells showed decreased proliferation, migration, and invasive capability, as well as a rounded cell shape. As ADSL is an enzyme involved in de novo purine synthesis, we first considered that the phenotypic changes in ADSL knockdown cells may have been due to decreased synthesis immunoblotting of HEC1B, HEC108, and SNGM cells transfected with siADSL or siControl. p-Akt (Ser473) band intensities of siADSL cells relative to those of siControl cells were calculated after normalization to Akt level. All data are representative of two independent experiments. Data are shown as means $\pm \mathrm{SD}$. $* * * P<0.001$, Student's $t$-test

of nucleotides, such as AMP, ATP, and GTP. However, although we could not measure the cytoplasmic nucleotide levels in this study, it was recently reported that ADSL knockdown did not result in decreases in AMP, ADP, ATP, or GTP in pancreatic $\beta$ cells [27]. This report prompted us to explore a mechanism of ADSL involvement in tumor cell aggressiveness independent of nucleotide synthesis. Indeed, in ADSL knockdown cells, expression of KLRC3, which was recently reported to be associated with glioblastoma aggressiveness [25], was shown to be reduced, and silencing of KLRC 3 in endometrial cancer cells induced the same phenotype as that of ADSL, suggesting that the reduced tumor cell aggressiveness of ADSL knockdown cells was due to decreased expression of KLRC3.

KLRC3 is a member of the NKG2 group, which is expressed in natural killer cells and encodes a family of transmembrane proteins with a C-type lectin domain. Although KLRC3 was reported to form a complex with CD94 interacting with HLA-E [31], the precise function has not been elucidated. Recently, it was reported that KLRC3 resides intracellularly, and not on the cell surface, and forms a complex with CD94 and DAP12 [24]. DAP12 has an immunoreceptor tyrosine-based activation motif, which activates the PI3K-Akt signaling pathway [28-30]. These observations suggest that KLRC3 may affect intracellular signal transduction by interacting with DAP12. Indeed, ADSL knockdown cells in which KLRC3 expression was decreased showed lower Akt phosphorylation than control cells in the present study. This result suggests that KLRC3 may be involved in the Akt signaling pathway, and reduced expression of KLRC 3 in ADSL knockdown cells may contribute to decreased proliferation, migration, invasive capability, and changes in cell shape via the Akt signaling pathway. 


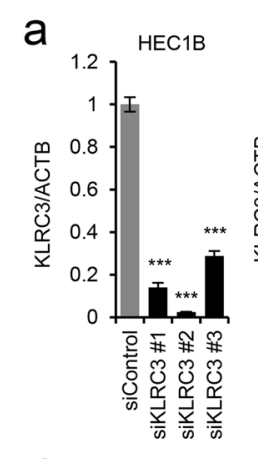

C
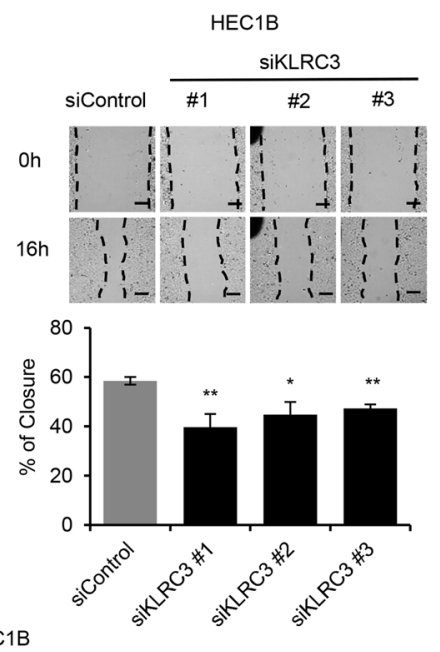

$\mathrm{d}_{\text {HEC1B }}$ sicontrol is is $_{\text {siKLRC3\#1 }}$
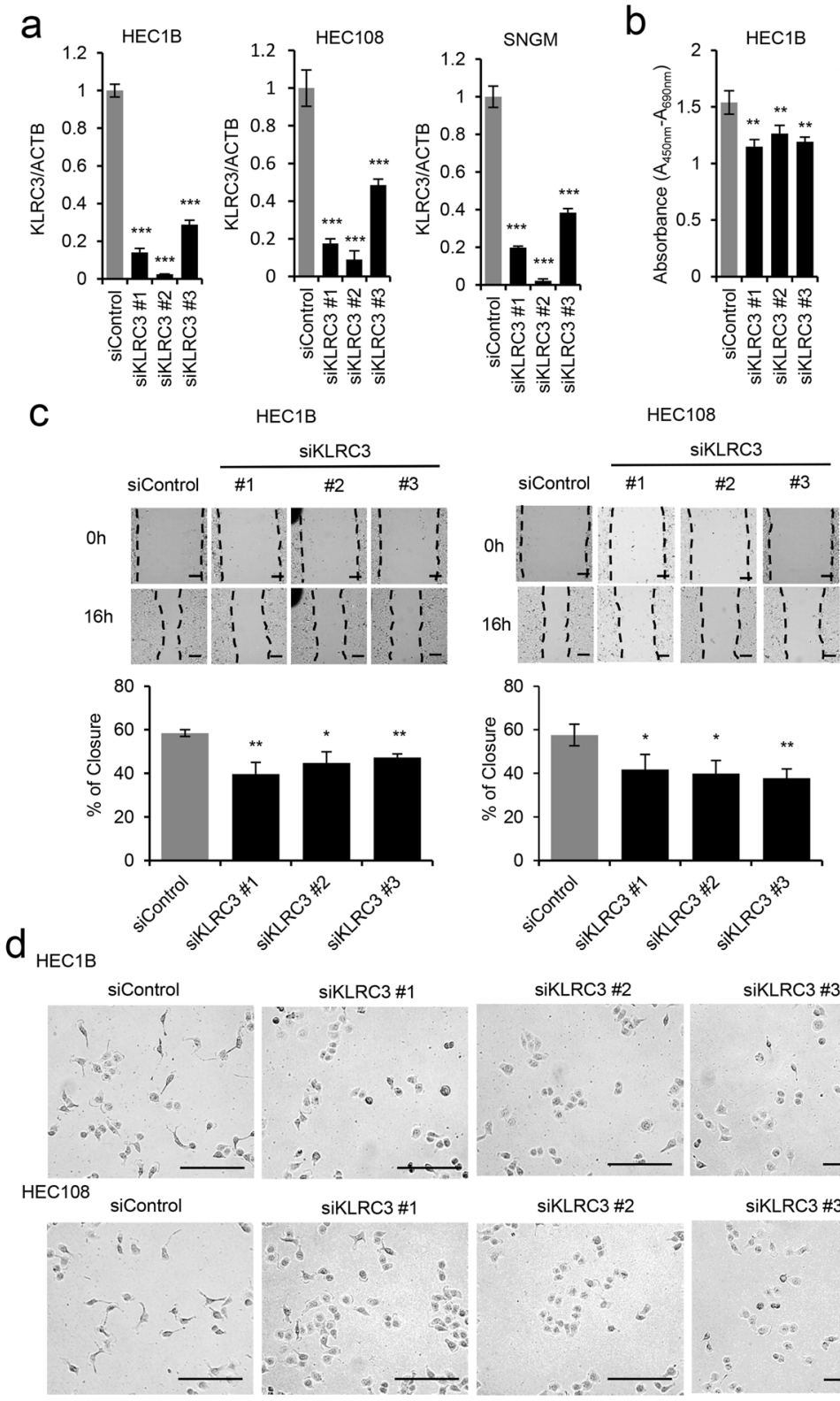

HEC108
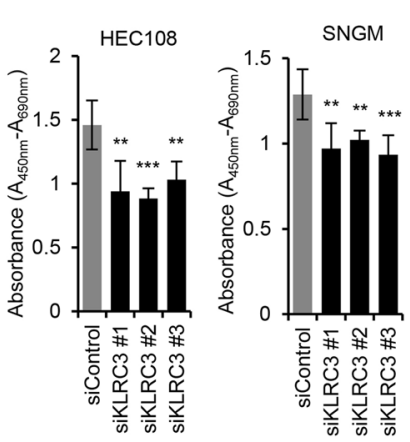

SNGM
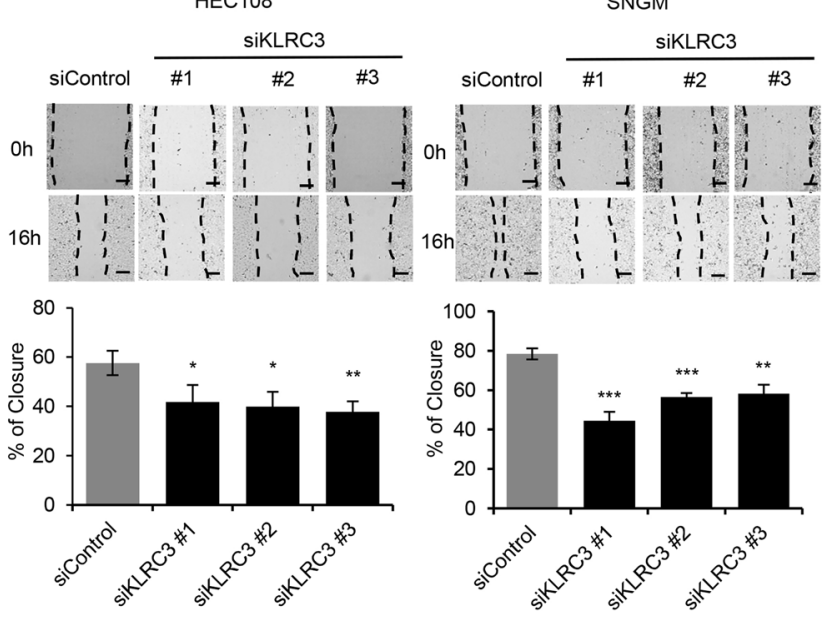

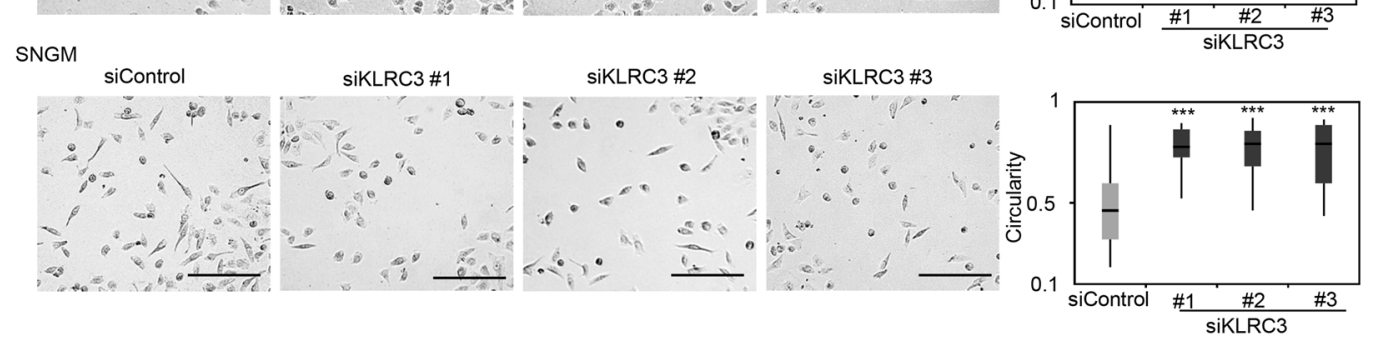

Fig. 5 KLRC3 knockdown decreases cell proliferation and migration capability. a The abundance of KLRC3 mRNA was evaluated by realtime PCR in HEC1B, HEC108, and SNGM cells transfected with three individual siRNA duplexes specific for KLRC3 (siKLRC3 \#1, \#2 and \#3) or non-targeting control siRNA (siControl). Actin beta (ACTB) mRNA abundance was used to normalize real-time PCR results. Data are expressed as a ratio relative to siControl cells. b WST-1 assay of HEC1B, HEC108, and SNGM cells transfected with siKLRC3 or siControl. c HEC1B, HEC108, and SNGM cells transfected with
siKLRC 3 or siControl were wounded (upper eight pictures). The ratios of the wound closing distance to the width at $0 \mathrm{~h}$ are shown in the lower graphs. d HEC1B, HEC108, and SNGM cells were imaged $72 \mathrm{~h}$ after transfection of siKLRC 3 or siControl (left four pictures). Circularity was measured using Image $\mathbf{J}$ software (right graphs). All data are representative of three independent experiments. Data are shown as means \pm SD in $\mathbf{a}-\mathbf{c}$. For box plots in $\mathbf{d}$, the box depicts the middle $50 \%$ of the records and the line indicates the median. Scale bars, $400 \mu \mathrm{m}$ in c, $100 \mu \mathrm{m}$ in d. ${ }^{*} P<0.05,{ }^{*} P<0.01,{ }^{*} * * P<0.001$, Student's $t$-test 
Fig. 6 Fumarate increases KLRC3 expression in ADSL knockdown cells. a Schematic representation of the reaction path in which ADSL produces fumarate by degrading SAICAR and S-AMP. $\mathbf{b}$ The abundance of $K L R C 3$ mRNA was evaluated by real-time PCR in HEC1B, HEC108, and SNGM cells transfected with siADSL or siControl and treated with 100 $\mu \mathrm{M}$ diethyl fumarate or DMSO for $72 \mathrm{~h}$. Actin beta (ACTB) mRNA abundance was used to normalize real-time PCR results. Data are expressed as a ratio relative to siControl cells treated with DMSO. c HEC1B,

HEC108, and SNGM cells transfected with siADSL or siControl were imaged after incubation with $100 \mu \mathrm{M}$ diethyl fumarate or DMSO for $72 \mathrm{~h}$ (left five pictures). Circularity was measured using Image $\mathbf{J}$ software (right graphs). d HEC1B, HEC108, and SNGM cells transfected with siADSL or siControl were wounded and the culture media were changed to the ones containing $100 \mu \mathrm{M}$ diethyl fumarate or DMSO after wounded (left 10 pictures). The ratios of the wound closing distance to the width at $0 \mathrm{~h}$ are shown in the right graphs. All data are representative of two independent experiments. Data are shown as means \pm SD in $\mathbf{b}$ and $\mathbf{d}$. For box plots in $\mathbf{c}$, the box depicts the middle $50 \%$ of the records and the line indicates the median. Scale bars, $100 \mu \mathrm{m}$ in c, $400 \mu \mathrm{m}$ in d. $* P<0.05$, $* * P<0.01, * * * P<0.001$,

Student's $t$-test a

b

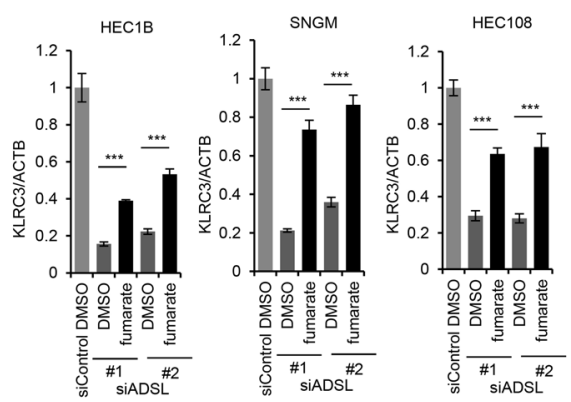

C
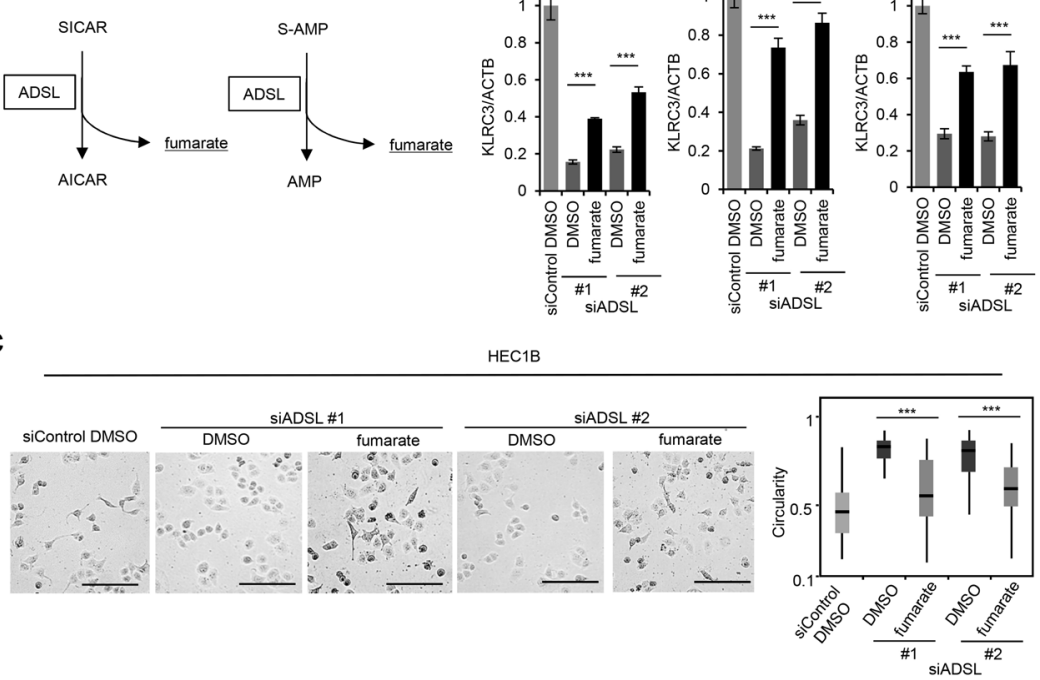

HEC10
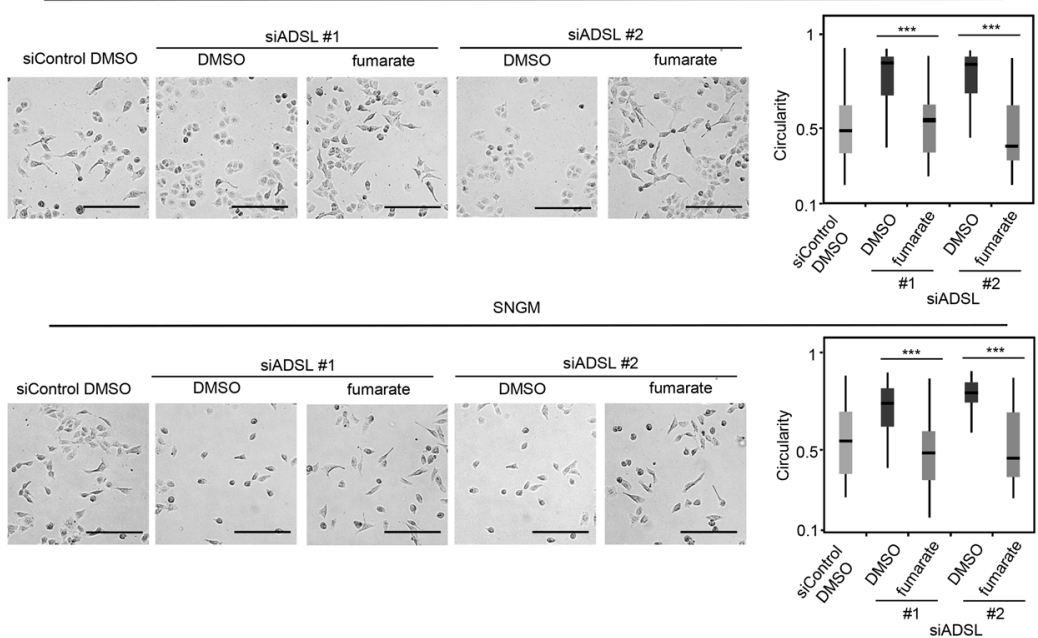

d
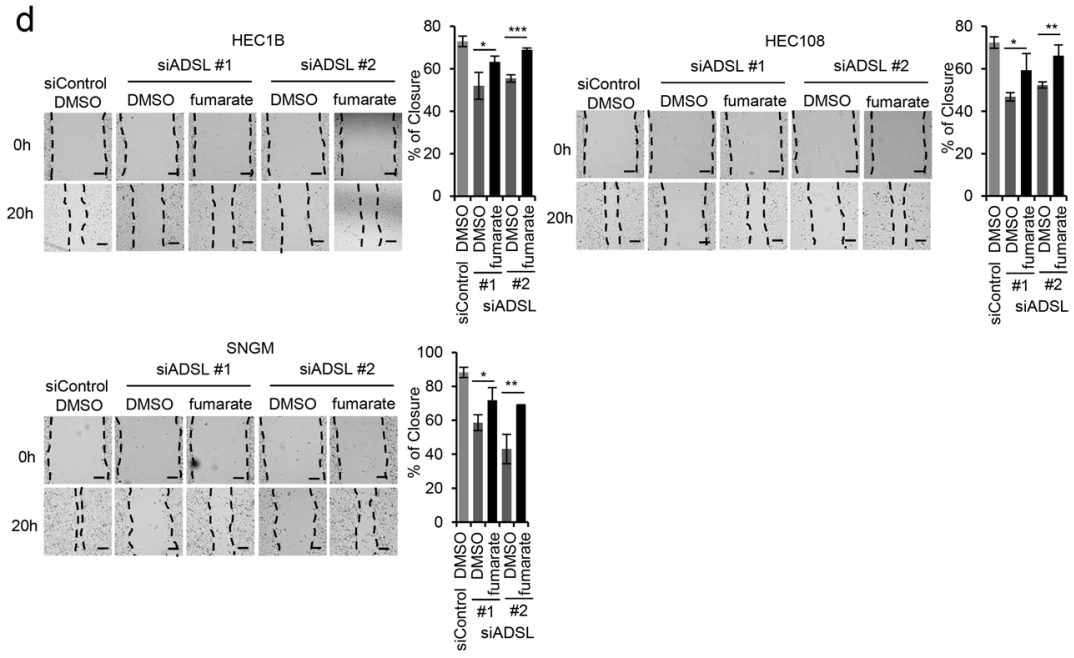
Finally, we showed that fumarate enhanced KLRC3 expression. Fumarate was recently shown to be an oncometabolite, regulating the expression of various genes through epigenetic changes and activating oncogenic pathways [17-20]. ADSL produces fumarate through the degradation of SAICAR and S-AMP. It was speculated that ADSL knockdown cells had lower levels of fumarate in the cytoplasm than control cells, and the decreased expression of KLRC 3 in ADSL knockdown cells may be due to reduced fumarate levels in the cytoplasm by silencing of ADSL. Indeed, exogenous fumarate recovered KLRC3 expression in ADSL knockdown cells. Moreover, increased expression of KLRC3 by adding fumarate to ADSL knockdown cells could eliminate the possibility that decreased KLRC3 expression in ADSL knockdown cells was due to off-target effects of ADSL silencing.

Recently, it was reported that fumarate inhibits $\alpha$ ketoglutarate-dependent dioxygenases that are involved in DNA and histone demethylation [21, 22]. Fumarate induces the EMT by inhibiting TET-mediated demethylation of the regulatory region of $m i R-200$ [23]. In addition, fumarate was reported to not only inhibit the function of TETs but also to decrease the expression levels of TET1 and TET2 [21]. In this study, however, TET1 transcript abundance was equivalent in ADSL knockdown and control cells (Supplementary Figure 2). In addition, the levels of EMTrelated gene expression by microarray analysis were the same in ADSL knockdown and control cells (Supplementary Table 1). These results suggest that the mechanism of decreased tumor cell aggressiveness in ADSL knockdown cells is independent of the TET-EMT pathway. This is consistent with the lack of correlations between ADSL expression and $\mathrm{N}$ grade in clinical human endometrioid carcinoma specimens, although more cases may be needed to be evaluated by immunohistochemistry to make a precise conclusion. We consider that KLRC 3 is the key factor in the mechanism by which ADSL knockdown cells showed decreased tumor cell aggressiveness, and the phenotypic changes in these cells were due to fumarate-dependent alterations in KLRC3 expression.

In conclusion, we have demonstrated that ADSL is involved in tumor cell proliferation, migration, invasive capability, and cell shape reorganization via regulation of KLRC3 expression by fumarate possibly produced by ADSL in endometrial cancer. These findings provide novel evidence for the contributions of ADSL and KLRC3 to tumor cell aggressiveness. Thus, ADSL and KLRC3 are potential new targets for the development of novel therapies for endometrial cancer.

Acknowledgements We thank Ms. Etsuko Maeno, Ms. Takako Sawamura, Ms. Megumi Nihei, and Mr. Masaharu Kohara for their technical assistance. This work was supported in part by JSPS
KAKENHI grant number T264604700, T254604350, T15K084230, and T15K083630.

\section{Compliance with ethical standards}

Conflict of interest The authors declare that they have no conflict of interest.

\section{References}

1. Jemal A, Siegel R, Ward E, et al. Cancer statistics, 2009. CA Cancer J Clin. 2009;59:225-49.

2. Chute JP, Muramoto GG, Whitesides J, et al. Inhibition of aldehyde dehydrogenase and retinoid signaling induces the expansion of human hematopoietic stem cells. Proc Natl Acad Sci USA. 2006;103:11707-12.

3. Ginestier C, Hur MH, Charafe-Jauffret E, et al. ALDH1 is a marker of normal and malignant human mammary stem cells and a predictor of poor clinical outcome. Cell Stem Cell. 2007;1:555-67.

4. Jiang F, Qiu Q, Khanna A, et al. Aldehyde dehydrogenase 1 is a tumor stem cell-associated marker in lung cancer. Mol Cancer Res. 2009;7:330-8.

5. Matsui W, Wang Q, Barber JP, et al. Clonogenic multiple myeloma progenitors, stem cell properties, and drug resistance. Cancer Res. 2008;68:190-7.

6. Pearce DJ, Taussig D, Simpson C, et al. Characterization of cells with a high aldehyde dehydrogenase activity from cord blood and acute myeloid leukemia samples. Stem Cells. 2005;23: 752-60.

7. Li T, Su Y, Mei Y, et al. ALDH1A1 is a marker for malignant prostate stem cells and predictor of prostate cancer patients' outcome. Lab Invest. 2010;90:234-44.

8. Rahadiani N, Ikeda J, Mamat S, et al. Expression of aldehyde dehydrogenase 1 (ALDH1) in endometrioid adenocarcinoma and its clinical implications. Cancer Sci. 2011;102:903-8.

9. Tahara S, Nojima S, Ohshima K, et al. S100A4 accelerates the proliferation and invasion of endometrioid carcinoma and is associated with the "MELF" pattern. Cancer Sci. 2016;107:1345-52.

10. Bridger WA, Cohen LH. The kinetics of adenylosuccinate lyase. J Biol Chem. 1968;243:644-50.

11. Reed VL, Mack DO, Smith LD. Adenylosuccinate lyase as an indicator of breast and prostate malignancies: a preliminary report. Clin Biochem. 1987;20:349-51.

12. Bardot V, Dutrillaux AM, Delattre JY, et al. Purine and pyrimidine metabolism in human gliomas: relation to chromosomal aberrations. Br J Cancer. 1994;70:212-8.

13. Terzuoli L, Carlucci F, Martino AD, et al. Determination of p185 and adenylosuccinate lyase (ASL) activity in preneoplastic colon lesions and intestinal mucosa of human subjects. Clin Biochem. 1998;31:523-8.

14. van Belzen N, Dinjens WN, Eussen BH, et al. Expression of differentiation-related genes in colorectal cancer: possible implications for prognosis. Histol Histopathol. 1998;13: 1233-42.

15. van Belzen N, Diesveld MP, van der Made AC, et al. Identification of mRNAs that show modulated expression during colon carcinoma cell differentiation. Eur J Biochem. 1995;234:843-8.

16. Wei S, Reillaudou M, Apiou F, et al. Purine metabolism in two human melanoma cell lines: relation to proliferation and differentiation. Melanoma Res. 1999;9:351-9.

17. Tomlinson IP, Alam NA, Rowan AJ, et al. Germline mutations in FH predispose to dominantly inherited uterine fibroids, skin leiomyomata and papillary renal cell cancer. Nat Genet. 2002;30:406-10. 
18. Isaacs JS, Jung YJ, Mole DR, et al. HIF overexpression correlates with biallelic loss of fumarate hydratase in renal cancer: novel role of fumarate in regulation of HIF stability. Cancer Cell. 2005;8:143-53.

19. Pollard PJ, Briere JJ, Alam NA, et al. Accumulation of Krebs cycle intermediates and over-expression of HIF1alpha in tumours which result from germline $\mathrm{FH}$ and SDH mutations. Hum Mol Genet. 2005;14:2231-9.

20. Yang M, Soga T, Pollard PJ, et al. The emerging role of fumarate as an oncometabolite. Front Oncol. 2012;2:85.

21. Laukka T, Mariani CJ, Ihantola T, et al. Fumarate and succinate regulate expression of hypoxia-inducible genes via TET enzymes. J Biol Chem. 2016;291:4256-65.

22. Xiao M, Yang $\mathrm{H}, \mathrm{Xu} \mathrm{W}$, et al. Inhibition of alpha-KG-dependent histone and DNA demethylases by fumarate and succinate that are accumulated in mutations of $\mathrm{FH}$ and $\mathrm{SDH}$ tumor suppressors. Genes Dev. 2012;26:1326-38.

23. Sciacovelli M, Goncalves E, Johnson TI, et al. Fumarate is an epigenetic modifier that elicits epithelial-to-mesenchymal transition. Nature. 2016;537:544-7.

24. Orbelyan GA, Tang F, Sally B, et al. Human NKG2E is expressed and forms an intracytoplasmic complex with CD94 and DAP12. J Immunol. 2014;193:610-6.
25. Cheray M, Bessette B, Lacroix A, et al. KLRC3, a natural killer receptor gene, is a key factor involved in glioblastoma tumourigenesis and aggressiveness. J Cell Mol Med. 2017;21:244-53.

26. Ohshima K, Nojima S, Tahara S, et al. Argininosuccinate synthase 1-deficiency enhances the cell sensitivity to arginine through decreased DEPTOR expression in endometrial cancer. Sci Rep. 2017;7:45504.

27. Gooding JR, Jensen MV, Dai X, et al. Adenylosuccinate is an insulin secretagogue derived from glucose-induced purine metabolism. Cell Rep. 2015;13:157-67.

28. Turnbull IR, Colonna M. Activating and inhibitory functions of DAP12. Nat Rev Immunol. 2007;7:155-61.

29. Craxton A, Jiang A, Kurosaki T, et al. Syk and Bruton's tyrosine kinase are required for $\mathrm{B}$ cell antigen receptor-mediated activation of the kinase Akt. J Biol Chem. 1999;274:30644-50.

30. Jiang K, Zhong B, Gilvary DL, et al. Syk regulation of phosphoinositide 3-kinase-dependent NK cell function. J Immunol. 2002;168:3155-64.

31. Kaiser BK, Barahmand-Pour F, Paulsene W, et al. Interactions between NKG2x immunoreceptors and HLA-E ligands display overlapping affinities and thermodynamics. J Immunol. 2005; 174:2878-84. 